\title{
Correlation between histopathological grading and shear-wave elastography in evaluating invasive carcinoma of no special type
}

\author{
YI-CHENG ZHU ${ }^{1}$, YUAN ZHANG ${ }^{1}$, SHU-HAO DENG ${ }^{1}$, QUAN JIANG ${ }^{1}$ and DENG-SHAN WANG ${ }^{2}$ \\ Departments of ${ }^{1}$ Ultrasound and ${ }^{2}$ Pathology, Pudong New Area People's Hospital Affiliated to \\ Shanghai University of Medicine and Health Sciences, Shanghai 201200, P.R. China
}

Received May 5, 2018; Accepted September 6, 2018

DOI: $10.3892 /$ etm.2018.6790

\begin{abstract}
The present study aimed to investigate the correlation between histologic grade and shear-wave elastography in evaluating invasive breast carcinoma of no special type (NST). A total of 84 breast lesions in 80 patients (age range, 32-64 years) were retrospectively evaluated. The patients underwent B-mode ultrasound and virtual touch tissue quantification diagnosis. A semi-quantitative method was then applied in order to determine the histologic grade of the invasive carcinoma of NST according to the grading system published by the World Health Organization. Among the 84 breast lesions, $14(16.7 \%)$ were classified as Grade I, while 29 (34.5\%) and 41 (48.8\%) were determined to be of Grade II and Grade III, respectively. The size of the breast lesions increased with the increase in histological grade $(\mathrm{P}<0.001)$. In addition, $29.3 \%$ of the Grade-III breast lesions exhibited acoustic enhancement, while the majority of the Grade-I breast lesions (78.6\%) exhibited acoustic shadowing $(\mathrm{P}=0.002)$. Furthermore, a higher histopathological grade was closely correlated with a higher mean, minimum and maximum shear-wave velocity value $(\mathrm{P}<0.05)$. In conclusion, regarding the evaluation of invasive breast carcinoma of NST, the histologic grade was strongly correlated with tissue stiffness; a higher histologic grade was associated with a harder lesion. Therefore, shear-wave elastography may provide important clinical reference values.
\end{abstract}

\section{Introduction}

Breast carcinoma is the most common cause of cancer-associated mortality in females and accounts for $6.9 \%$ of all cancer-associated mortalities in Chinese females (1). The majority of breast cancer cases are defined

Correspondence to: Mr. Yuan Zhang, Department of Ultrasound, Pudong New Area People's Hospital Affiliated to Shanghai University of Medicine and Health Sciences, 490 South Chuanhuan Road, Shanghai 201200, P.R. China

E-mail: Yuan_ZMD@126.com

Key words: invasive ductal carcinoma, shear-wave elastography, histologic grading, ultrasonography, breast neoplasms as invasive carcinoma of no special type (NST) (2). The global five-year survival rate of invasive carcinoma of NST after diagnosis is $70-90 \%$ (3). Due to favorable prognosis, detection of breast carcinoma at the early stage is crucial for successful clinical treatment. Ultrasound (US) is a widely accepted technique for differentiating between benign and malignant breast lesions (BLs). In addition to B-mode US, elastography has been developed to aid clinicians in the assessment of tissue stiffness. A previous study demonstrated that malignant lesions tended to be harder, while softer lesions comprised the bulk of benign lesions (4). In the case of invasive breast carcinoma, disordered collagen makes BLs poorly compressible. Conversely, even if benign BLs, e.g., fibroadenoma, are rich in collagen, they are commonly less stiff due to the well-ordered collagen. In addition, a previous study indicated that the histopathological classification has a direct impact on the five-year survival rate of females with invasive carcinoma of NST (3).

The histopathological classification has a direct impact on the therapeutic effect and prognosis. However, to the best of our knowledge, few studies have reported on the association between elastography and histopathology in breast cancers, particularly in invasive carcinoma of NST. Therefore, the present study aimed to investigate the correlation between the classification based on pathologic histology and shear-wave elastography in evaluating invasive carcinoma of NST.

\section{Patients and methods}

Clinical data. The present retrospective study involved 259 patients who had undergone conventional US at Pudong New Area People's Hospital (Shanghai, China) between March 2016 and February 2018 and in whom BLs were detected. The Ethical Committee of Pudong New Area People's Hospital approved the retrospective study of the images and associated records of the enrolled patients. All data were reported anonymously. Informed consent was obtained for the publication of any images. In total, $84 \mathrm{BLs}$ in 80 patients (age range, 32-64 years; mean age, $46.87 \pm 7.82$ years) were selected for the present study. The selection flowchart is presented in Fig. 1, with the following inclusion criteria: i) BL was detectable by ultrasound; ii) BLs were pathologically confirmed as invasive carcinoma of NST; iii) complete pathological records were available; iv) the BL that was 
examined had no history of treatment. The mean diameter of the lesions was $24.47 \pm 10.09 \mathrm{~mm}$ (range, 10.2-48.3 $\mathrm{mm}$ ) and the mean depth was $24.93 \pm 11.30 \mathrm{~mm}$ (range, 8.1-47.3 mm).

Apparatus and methods. The patients first underwent B-mode US in the supine position. An Acuson S2000 ${ }^{\mathrm{TM}}$ Ultrasound System (Siemens Healthcare, Munich, Germany), equipped with a 9L4 linear transducer $(4-9 \mathrm{MHz}, 4.0 \mathrm{~cm})$ was used. One radiologist (Y-CZ), who had $>3$ years of experience in breast sonography and had 4 months of experience in virtual touch tissue quantification (VTQ) measurement, performed the B-mode US examination. Once a lesion was detected, the radiologist recorded a number of basic ultrasonic features, including lesion size in diameter, lesion depth (vertical distance from the skin to the bottom of the lesion), margin and echotexture. Following the examination, the patients then underwent shear-wave elastography, specifically VTQ diagnosis, using the same machine. The same operator further observed the elasticity of the BL using VTQ by reading the same imaging area of the B-mode US. The transducer was strapped to the patient's chest lightly and patient was asked to hold their breath for several seconds. The radiologist measured the shear-wave velocity (SWV) of the lesion and the normal breast tissues by using a quantification region of interest (ROI) box (with fixed dimensions of $5 \times 6 \mathrm{~mm}$ ). The ROI box on the shear-wave velocity (SWV) image included the mass and surrounding breast tissue. Visible calcifications and cystic components were avoided as far as possible and the ROI was placed in the solid region during the investigation. The SWV values were obtained from the ROI in the solid portion of the mass and the surrounding breast tissue at the same depth. Absolute measurements of SWV, displayed in meters per second, were generated by evaluating the peak displacement at each transverse. The same depth, focus position and gain setting were used for the same lesion. The operator performed the measurement using the same methodology for seven times. The rational of repeating the same methodology seven times was the following: The rapid malignant proliferation led to insufficient blood supply and eventually resulted in localized liquefaction and necrosis. This further induced the stiffness of the breast tissue. A slower SWV was associated with softer BLs. Furthermore, the entire lesion did not exhibit a consistent hardness due to the inhomogeneous proliferation rates of the cancer cells. Proliferation increased fibrosis and decrease necrosis, thus areas with high proliferation rates were stiffer compared with those with low proliferation rates (5). Therefore, it was necessary to repeat the same process for seven times. The SWV values and the percentage of successful measurements were recorded and examined. The scale for the SWV was $0-9.1 \mathrm{~m} / \mathrm{sec}$.

In terms of pathological examination, one pathologist with 10 years of experience (DW) applied a semi-quantitative method to classify invasive carcinoma of NST according to the grading system developed by the World Health Organization (WHO) (6). A numerical scoring system with a scale of 1-3 was used to assess three features in total, namely tubule and gland formation, nuclear pleomorphism and mitotic counts. The cut-off points for the first feature were 75 and 10 for score allocation: One point was assigned on the condition that $>75 \%$ of the mass area consisted of definite tubules, and tumors that exhibited between 10 and $75 \%$ tubule formation were scored as 2 points. With regard to nuclear pleomorphism, a score of 1 referred to those small, regular uniform cells that were highly similar in size with that of the benign pre-existing epithelial cells. A score of 2 was assigned for mild to moderate pleomorphism and inconspicuous nucleoli, while a score of 3 demonstrated that the nuclei were larger in size with marked variation. Mitotic counts were determined as the number of mitotic figures observed in 10 consecutive high-power fields $(0.42 \mathrm{~mm})$ in the most mitotically active part of the tumor (the leading edge). The cut-off points for this characteristic were 5 and 10: The presence of 0-5 mitotic figures was rated as $1 ; 6-10$ mitotic figures were scored as 2 and $>10$ mitotic figures were scored as 3. A final grading was calculated as the sum of the three aforementioned values (7). Grade I (well-differentiated) referred to those with a score of 3-5, Grade II (moderately differentiated) was assigned to those with a score of 6 or 7 , and those with a score of $>7$ were rated as Grade III (poorly differentiated).

Statistical analysis. The Chi-square test or Fisher's exact test were applied in order to evaluate differences between groups in terms of categorical variables, while analysis of variance was used to compare continuous variables between the groups. $\mathrm{P}<0.05$ was considered to indicate a statistically significant difference. All data were analyzed using SPSS 25.0 (IBM Corp., Armonk, NY, USA).

\section{Results}

Ultrasonic features. Of the 84 BLs, 14 (16.7\%) were rated as Grade I, 29 (34.5\%) as Grade II and 41 (48.8\%) as Grade III. The results demonstrated that there were three ultrasonic characteristics that were significantly associated with the histological grade (Table I). First, the size of the BL increased with the increase in histological grade $(\mathrm{P}<0.001)$. The median diameter of the Grade-I BLs was $12.90 \pm 0.59 \mathrm{~mm}$, whilst the size of the Grade III BLs was twice of that $(25.90 \pm 1.56 \mathrm{~mm})$. Furthermore, $29.3 \%$ of the Grade-III BLs exhibited acoustic enhancement, while the majority of the Grade-I BLs (78.6\%) exhibited acoustic shadowing $(\mathrm{P}=0.002)$. Finally, the Grade-III BLs tended to display acoustic enhancement; however, this was relatively rare in the Grade-I and Grade-II BLs (7.1 and $3.4 \%$, respectively). In total, 2 out of 43 (4.7\%) Grade-I and Grade-II BLs exhibited acoustic enhancement, whereas 15 out of $41(36.6 \%)$ Grade-III BLs exhibited the same feature $(\mathrm{P}=0.002)$. Other ultrasonic features of the invasive carcinoma of NST of different grades, including the margin, echotexture and halo sign, were not significantly associated with the histopathologic classification (all $\mathrm{P}>0.05$ ).

Association of VTQ results with the histopathological grading. The results demonstrated that a higher histopathological grade was closely associated with a higher minimum, mean and maximum SWV value (Figs. 2 and 3). The Grade-III BLs exhibited the highest mean SWV value of $6.32 \mathrm{~m} / \mathrm{sec}$ [interquartile range (IQR), 5.35-6.98 m/sec], 
Table I. Ultrasonic features of all of the invasive carcinomas of NST of different histopathological grades.

\begin{tabular}{|c|c|c|c|c|}
\hline Characteristic & Grade I $(n=14)$ & Grade II $(n=29)$ & Grade III $(n=41)$ & P-value \\
\hline Age (years) & $49.57 \pm 8.15$ & $48.59 \pm 7.99$ & $44.73 \pm 7.15$ & 0.045 \\
\hline Position & & & & 0.605 \\
\hline Right & $8(57.1)$ & $12(41.4)$ & $18(43.9)$ & \\
\hline Left & $6(42.9)$ & $17(58.6)$ & $23(56.1)$ & \\
\hline Lesion size (mm) & $12.90(10.90-15.65)$ & $22.90(18.25-31.9)$ & $25.90(19.25-34.85)$ & $<0.001$ \\
\hline Margin & & & & 0.089 \\
\hline Well-defined & $0(0.0)$ & $2(6.9)$ & $8(19.5)$ & \\
\hline Poorly defined & $14(100.0)$ & $27(93.1)$ & $33(80.5)$ & \\
\hline Echotexture & & & & 0.944 \\
\hline Heterogeneous & $10(71.4)$ & $22(75.9)$ & $31(75.6)$ & \\
\hline Homogeneous & $4(28.6)$ & $7(24.1)$ & $10(24.4)$ & \\
\hline Halo sign & & & & 0.692 \\
\hline Present & $4(28.6)$ & $11(37.9)$ & $17(41.5)$ & \\
\hline Absent & $10(71.4)$ & $18(62.1)$ & $24(58.5)$ & \\
\hline Calcification & & & & 0.443 \\
\hline No & $6(42.9)$ & $11(37.9)$ & $11(26.8)$ & \\
\hline Yes & $8(57.1)$ & $18(62.1)$ & $30(73.2)$ & \\
\hline Posterior acoustic feature & & & & 0.002 \\
\hline None & $2(14.3)$ & $7(24.1)$ & $7(17.1)$ & \\
\hline Shadowing & $11(78.6)$ & $14(48.3)$ & $12(29.3)$ & \\
\hline Enhancement & $1(7.1)$ & $1(3.4)$ & 15 (36.6) & \\
\hline Mixed & $0(0.0)$ & $7(24.1)$ & $7(17.1)$ & \\
\hline
\end{tabular}

Values are expressed as $\mathrm{n}(\%)$, the median (interquartile range) or the mean \pm standard deviation. NST, no special type.

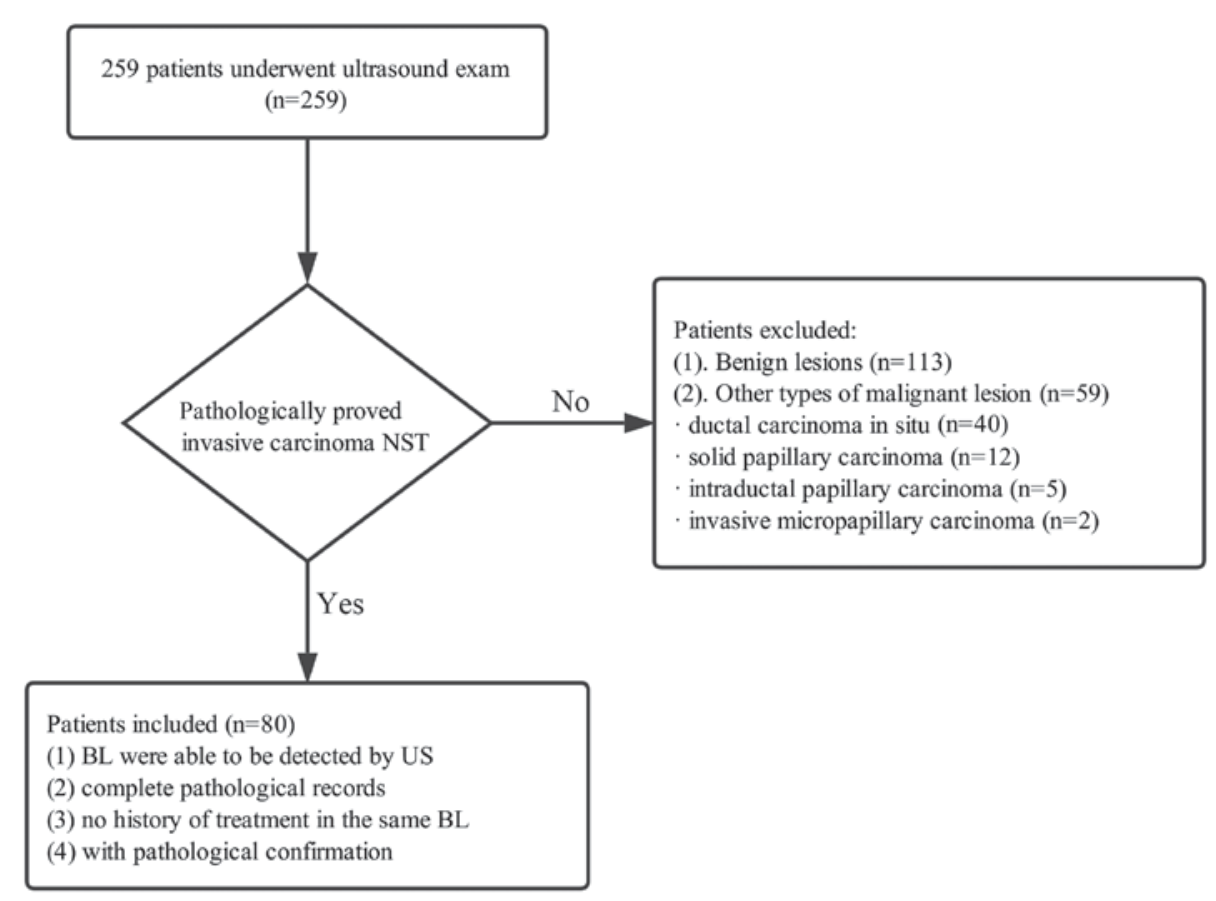

Figure 1. Flowchart of patient selection. BL, breast lesion; NST, no special type; US, ultrasound.

followed by those of Grade II (mean, $4.37 \mathrm{~m} / \mathrm{sec}$; IQR, 2.56-4.54 m/sec; P=0.032). However, the minimum, mean $3.98-4.58 \mathrm{~m} / \mathrm{sec}$ ) and Grade I (mean, $4.31 \mathrm{~m} / \mathrm{sec}$; IQR, and maximum SWV values were not significantly associated 


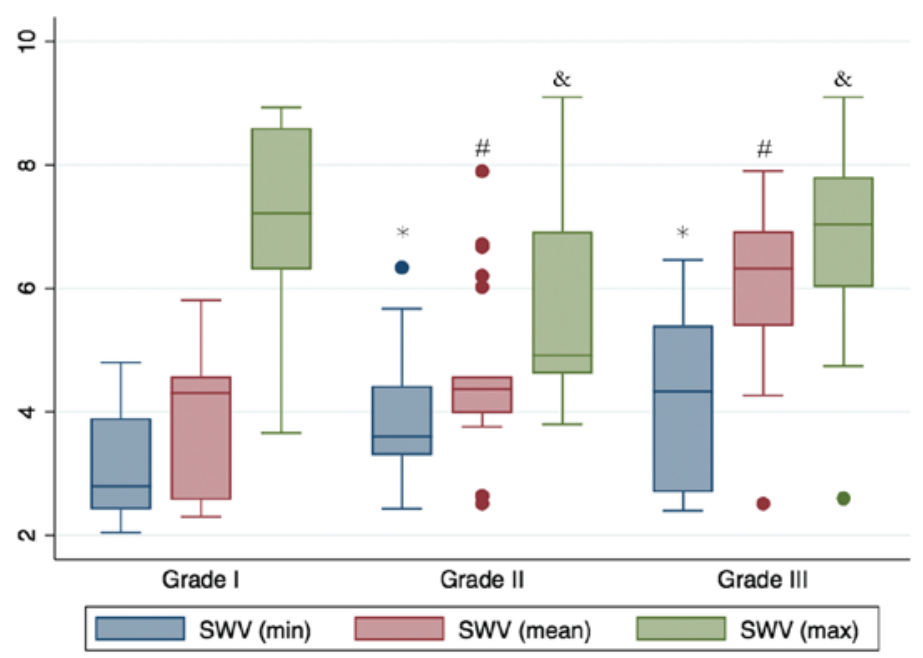

Figure 2. Boxplot of SWV values for breast lesions of different histologic grades. The box plot displays boxes bordered by the 25th and 75th percentiles with a median line at the 50th percentile; whiskers represent the upper and lower adjacent values. "P<0.05 vs. SWV (min) Grade I. "P<0.05 vs. SWV (mean) Grade I. ${ }^{\&} \mathrm{P}<0.05$ vs. SWV (max) Grade I. SWV, shear-wave velocity.

A

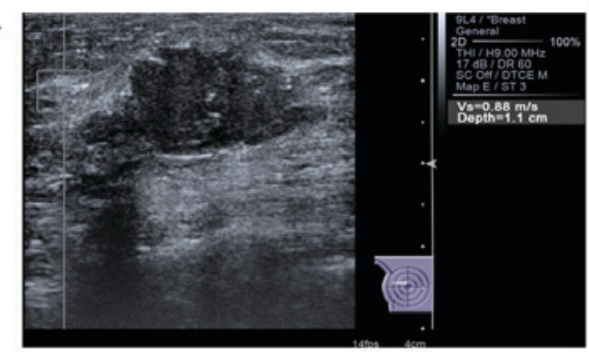

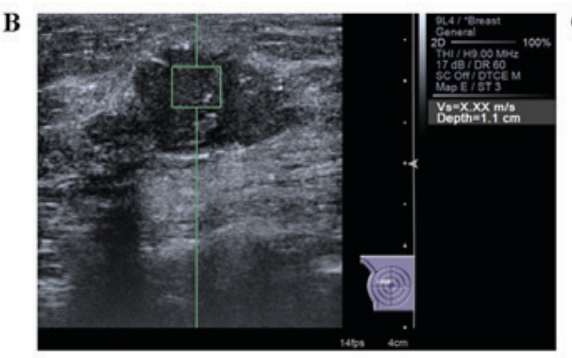

Figure 3. Evaluation of SWV values in a case of invasive carcinoma of no special type in the right breast of a 58-year-old female patient. (A) The SWV of the surrounding breast tissue was $0.88 \mathrm{~m} / \mathrm{sec}$, while (B) the SWV of the mass was X.XX m/sec. The non-applicable situation occurred when the screen displayed ' $\mathrm{X} . \mathrm{XX} \mathrm{m} / \mathrm{sec}$ ', which may be interpreted as the tissue being either extremely soft or hard as it exceeded the range of the SWV value. The value was then denoted as either 9.1 or $0 \mathrm{~m} / \mathrm{sec}$, depending on whether it was the solid portion or the cystic portion, respectively, once technical failures or other potential factors, including the patient's breathing, were ruled out. In this case, the two interpreters agreed to denote the value as $9.1 \mathrm{~m} / \mathrm{sec}$. (C) In terms of histologic grading, the lesion was classified as Grade II (H\&E; magnification, x100). SWV, shear-wave velocity.

with the lesion size (Table II). For instance, as a representative case, a 61-year-old female patient presented at Pudong New Area People's Hospital with a large palpable mass (data not shown). The 48.3-mm BL exhibited a high mitotic frequency and central necrosis, and was histologically classified as Grade III (data not shown). The BL was pathologically confirmed as pleomorphic carcinoma, a rare variant of high-grade invasive carcinoma of NST (data not shown).

\section{Discussion}

Although the morbidity of breast carcinoma and the mortality of affected patients are not excessively high among Chinese females, the incidence rate in China has been increasing over the past decades (8). Early diagnosis is vital for increasing the five-year survival rate and prognosis, particularly for females with invasive carcinoma of NST. According to the WHO classification, breast carcinomas may be classified into three histopathological grades. These grades have significance for prognostic implications, as breast cancer encompasses a great diversity of subtypes, grades, risk of metastasis and prognosis. The histopathological classification integrates three components that are crucial for breast cancer development. First, the cell morphology indicates pleomorphism of the nuclei. Nuclei vary in size and shape; larger and abnormal nuclei frequently have multiple nucleoli and moderate vesicular nuclei exhibit visible nucleoli. Second, the percentage of tubule formation is accepted for quantifying the proliferation. The third indicator is mitotic activity; the higher the mitotic activity, the more rapid the proliferation. This well-established grading system is applied for the unified evaluation of tumor differentiation together with additional objective criteria. The histopathological grade acknowledges molecular events that are detected in histological morphology, which further assists in the identification of breast carcinoma growth and thus the adoption of the most appropriate treatment. In general, high levels of proliferation are commonly observed in poorly-differentiated masses, while lower well-differentiated masses have lower proliferation indexes. A number of studies have confirmed the role of histological prognostic factors in guiding adequate therapies (9-13).

With regard to conventional ultrasonography, the results of the present study indicated that the histologic grade was highly associated with the lesion size and posterior acoustic features. First, a higher the histologic grade of a lesion was associated with a larger lesion size. The size of Grade-III BLs 
Table II. SWV values in correlation with histological grade and lesion size.

SWV

\begin{tabular}{lccc}
\cline { 2 - 4 } Value & Minimum & Mean & Maximum \\
\hline Histologic grade & & & $4.84(2.69-5.40)$ \\
1 & $3.43(2.43-4.03)$ & $4.31(2.56-4.54)$ & $4.84(4.56-5.70)$ \\
2 & $3.60(3.40-4.42)$ & $4.37(3.98-4.58)$ & $7.04(6.06-7.81)$ \\
3 & $5.60(4.62-6.10)$ & $6.32(5.35-6.98)$ & 0.032 \\
P-value & 0.011 & 0.032 & $7.37(6.20-8.60)$ \\
Size $(\mathrm{mm})$ & & & $5.24(4.37-6.38)$ \\
$<20$ & $3.30(2.43-4.43)$ & $4.71(3.76-6.72)$ & 0.482 \\
$\geq 20$ & $3.90(3.30-5.05)$ & $6.02(4.92-7.28)$ & 0.525 \\
P-value & 0.411 & & \\
\hline
\end{tabular}

Values are expressed as $\mathrm{Q}_{50}$ (interquartile range). SWV, shear-wave velocity.

was nearly twice of that of Grade-I lesions. Furthermore, the posterior acoustic characteristics were identified to be closely associated with the histologic grade of the lesion. BLs that exhibited acoustic shadowing were more commonly identified in patients with grade I and II invasive carcinoma of NST. In total, $78.6 \%$ of the Grade-I BLs and $48.3 \%$ of the Grade-II BLs, buy only $29.3 \%$ of the Grade-III BLs featured acoustic shadowing. This result suggests that different histological grades of BLs had distinct attenuation characteristics. In the lesions that displayed acoustic shadowing, a clear growth of fibrous tissue was observed and this growth was normally accompanied with sclerotic stroma. This may be the reason why invasive carcinoma of NST often presents with a scirrhous and stellate appearance. Blaichman et al (14) suggested a strong link between posterior acoustics and histologic grade after reviewing 299 invasive breast carcinomas of NST of Breast Imaging Reporting and Data System 4 and 5. The results indicated that only 13 out of 108 Grade-III BLs (12.0\%) exhibited acoustic shadowing, while 22 (57.9\%) and 67 (43.8\%) of Grade-I and -II BLs, respectively, had the same characteristic. The Grade-III BLs had a greater tendency to display posterior enhancement $(53 / 108 ; 49.1 \%)$. In addition, the results of the present study were consistent with those of Rotstein and Neerhut (15), who determined that high-grade invasive ductal carcinomas were less prone to exhibiting acoustic shadowing compared to low- and intermediate-grade carcinomas. The amount of connective tissue volume was suggested to be one of the factors that influences acoustic shadowing. Scirrhous carcinomas frequently consist of rich connective tissues, which bring about attenuation in neoplastic tissue and therefore cause acoustic shadowing. Furthermore, attenuation is also determined by tissue organization, as reported by Gozzi et al (16). With regard to acoustic enhancement, Blaichman et al (14) identified that the cellular content was proportional to acoustic enhancement. Higher-grade BLs demonstrated a tendency to exert marked desmoplastic effects on peritumoral tissues, which led to intensive cellularity (14). Therefore, Grade-III BLs have a higher potential to exhibit posterior enhancement $(36.6 \%$ of cases in the present study), whereas intermediate- and low-grade BLs have a higher probability of exhibiting posterior shadowing (48.3 and $78.6 \%$ of cases in the present study, respectively).

The VTQ technique is a quantitative tool for evaluating tissue stiffness. The basic working principle is shear-wave propagation, which is induced by a specific acoustic impulse. Advanced VTQ is able to directly and mechanically detect the velocities of transverse waves to provide quantified information on tissue deformations. The SWV is therefore generated through interference of waves with the tissue and is proportional to tissue stiffness. In contrast to static elastography, absolute SWV values expressed in $\mathrm{m} / \mathrm{sec}$ are obtained by evaluating the peak displacement at each transverse wave. A number of studies have evaluated the diagnostic performance of the measurement of quantitative elasticity values of thyroid nodules (17), metastatic and non-metastatic cervical lymph nodes (18) and acute pancreatitis (19). The elastographic standard deviation was rated as a valuable means of measuring heterogeneity in the aforementioned studies. Heterogeneity has been widely accepted as a crucial indicator in distinguishing between benign and malignant lesions. In the case of evaluating breast masses, there is an increasing trend of applying VTQ measurement $(20,21)$. For instance, Tozaki et al (22) concluded that the mean SWV value of the malignant neoplasms was larger than that of benign masses $(\mathrm{P}<0.01)$. In addition, Golatta et al $(23)$ examined 104 BLs in 103 patients via VTQ and concluded that malignant lesions are stiffer than benign masses in terms of the mean maximum velocity. In the present study, the association between VTQ parameters and the histological grade of carcinoma of NST was investigated. The results indicated that invasive carcinoma of NST of a higher grade tend to be stiffer. Previous studies investigating the association between semi-quantitative elastography measurements and histopathological grading of invasive breast carcinoma have provided similar results $(24,25)$. In addition to the elastographic standard deviation, higher minimum and maximum SWV values were also obtained for higher-grade BLs in the present study. This result may be highly associated with regulatory factors. With the increasing histopathological grade, the mitotic figures became more active 
and the mitosis was more pronounced. In other words, the malignant proliferation index was higher. In the present study, it was demonstrated that higher-degree BLs exerted excessive desmoplastic effects on peritumoral tissues. Abundant cells conglomerated, which resulted in larger tension of the lesions and a greater increase in the synthesis of collagen fibers in the stroma. Consequently, a higher histopathological grade was associated with a stiffer lesion. In turn, in the high-grade BLs, the SWV value was greater than that of the lower-grade BLs.

The present study had a number of limitations. First, the sample size was not sufficiently large, as the study was performed at only one hospital. Therefore, further studies with a larger sample size are necessary to validate the present results. Furthermore, selection bias was not avoidable due to the retrospective nature of the study and future prospective study is required. Owing to its retrospective nature, the present study did not explore any associations between stiffness and other important tumor characteristics, including the vascularity of the lesions, which is considered as an important feature for increasing the diagnostic specificity.

In conclusion, tissue stiffness of invasive carcinoma of NST is highly associated with the histopathological grade. The higher the pathological grade, the stiffer the BL. Lesion size, posterior acoustic characteristics and tissue stiffness together with histopathological grade are important complementary prognostic factors for evaluating invasive carcinoma of NST. A better understanding of histopathological grading and tissue stiffness are essential in order to avoid unnecessary surgery. Therefore, shear-wave elastography may enhance the meaning of histological grading and provide an important clinical reference value.

\section{Acknowledgements}

Not applicable.

\section{Funding}

The present study was supported by Pu Dong New Area Health and Family Planning Commission Subject Leader Course Project (grant no. PWRd 2017-06) and Pu Dong New Area Health and Family Planning Commission Important Vulnerable Course Project (grant no. PWzbr 2017-10).

\section{Availability of data and materials}

The datasets used and/or analyzed during the current study are available from the corresponding author on reasonable request.

\section{Ethics approval and consent to participate}

The Ethics Committee of Shanghai Pudong New Area People's Hospital (Shanghai, China) approved the retrospective study of the images and associated records of the enrolled patients.

\section{Authors' contributions}

YCZ designed the study DSW ollected and analyzed the patients' data regarding pathology. SHD collected the patients' data regarding tissue stiffness. YZ, QJ and SHD interpreted the data. YCZ was a major contributor in writing the manuscript. All authors read and approved the final manuscript.

\section{Patient consent for publication}

Not applicable.

\section{Competing interests}

The authors declare that they have no competing interests.

\section{References}

1. Zuo TT, Zheng RS, Zeng HM, Zhang SW and Chen WQ: Female breast cancer incidence and mortality in China, 2013. Thorac Cancer 8: 214-218, 2017

2. Schatten H: Cell and molecular biology of breast cancer. Humana Press, 2013

3. McCart Reed AE, Kalaw E, Reid A and Lakhani SR: Breast Cancer-Pathology and Genetics. In: Reference Module in Biomedical Sciences. Elsevier, 2018.

4. Luo J, Cao Y, Nian W, Zeng X, Zhang H, Yue Y and Yu F: Benefit of shear-wave elastography in the differential diagnosis of breast lesion: A diagnostic meta-analysis. Med Ultrason 1: 43-49, 2018.

5. Chamming's F, Latorre-Ossa H, Le Frere-Belda MA, Fitoussi V, T, Quibel T, Assayag F, Marangoni E, Autret G, Balvay D, Pidial L, et al: Shear wave elastography of tumour growth in a human breast cancer model with pathological correlation. Eur Radiol 23: 2079-2086, 2013.

6. Lakhani SR, Ellis IO, Schnitt SJ, Tan PH and van de Vijver MJ: WHO Classification of Tumours of the Breast, 4th Ed., 2012.

7. Elston CW, and Ellis IO: Pathological prognostic factors in breast cancer I. The value of histological grade in breast cancer: Experience from a large study with long-term follow-up. Histopathology 41: 154-161, 2002.

8. Li T, Mello-Thoms C and Brennan PC: Descriptive epidemiology of breast cancer in China: Incidence, mortality, survival and prevalence. Breast Cancer Res Treat 159: 395-406, 2016.

9. Chang J, Clark GM, Allred DC, Mohsin S, Chamness G and Elledge RM: Survival of patients with metastatic breast carcinoma: Importance of prognostic markers of the primary tumor. Cancer 97: 545-553, 2003.

10. Weidner N, Cady B and Goodson III WH: Pathologic prognostic factors for patients with breast carcinoma: Which factors are important. Surg Oncol Clin N Am 6: 415-462, 1997.

11. Irshad A, Ackerman SJ, Pope TL, Moses CK, Rumboldt T and Panzegrau B: Rare breast lesions: Correlation of imaging and histologic features with who classification. Radiographics 28: 1399-1414, 2008

12. Wang WS, Hardesty L, Borgstede J, Takahashi J and Sams S: Breast cancers found with digital breast tomosynthesis: A comparison of pathology and histologic grade. Breast J 22: 651-656, 2016.

13. Kim TH, Kang DK, Kim JY, Han S and Jung Y: Histologic grade and decrease in tumor dimensions affect axillary lymph node status after neoadjuvant chemotherapy in breast cancer patients. J Breast Cancer 18: 394-399, 2015.

14. Blaichman J, Marcus JC, Alsaadi T, El-Khoury M, Meterissian S and Mesurolle B: Sonographic appearance of invasive ductal carcinoma of the breast according to histologic grade. Am J Roentgenol 199: 402-408, 2012.

15. Rotstein AH and Neerhut PK: Ultrasound characteristics of histologically proven grade 3 invasive ductal breast carcinoma. Australas Radiol 49: 476-479, 2005.

16. Gozzi G, Cressa C, Bazzocchi M, Stanta G and Vidali C: Causes of attenuation of the sound waves in neoplasms of the breast: Histologic and echographic correlation study (In Italian). Radiol Med 72: 195-198, 1986.

17. Zhang FJ, Han RL and Zhao XM: The value of virtual touch tissue image (VTI) and virtual touch tissue quantification (VTQ) in the differential diagnosis of thyroid nodules. Eur J Radiol 83: 2033-2040, 2014.

18. Zhao Y, Xi J, Zhao B, Xiong W, Jiang D, Yang L, Cai Z, Liu T, Jiang H, Rong S and Jin X: Preliminary evaluation of virtual touch tissue imaging quantification for differential diagnosis of metastatic and nonmetastatic cervical lymph nodes. J Ultrasound Med 36: 557-563, 2017. 
19. Onoyama T, Koda M, Fujise Y, Takata T, Kawata S, Okamoto T, Miyoshi K, Matono T, Sugihara T, Matsumoto K, et al: Utility of virtual touch quantification in the diagnosis of pancreatic ductal adenocarcinoma. Clin Imaging 42: 64-67, 2017.

20. Jin ZQ, Li XR, Zhou HL, Chen JX, Huang X, Dai HX, Li JW, Chen XD and Xu XH: Acoustic radiation force impulse elastography of breast imaging reporting and data system category 4 breast lesions. Clin Breast Cancer 12: 420-427, 2012.

21. Zhou BG, Wang D, Ren WW, Li XL, He YP, Liu BJ, Wang Q, Chen SG, Alizad A and Xu HX: Value of shear wave arrival time contour display in shear wave elastography for breast masses diagnosis. Sci Rep 7: 7036, 2017.

22. Tozaki M, Isobe S and Fukuma E: Preliminary study of ultrasonographic tissue quantification of the breast using the acoustic radiation force impulse (ARFI) technology. Eur J Radiol 80 e182-e187, 2011.

23. Golatta M, Schweitzer-Martin M, Harcos A, Schott S, Gomez C, Stieber A, Rauch G, Domschke C, Rom J, Schütz F, et al: Evaluation of virtual touch tissue imaging quantification, a new shear wave velocity imaging method, for breast lesion assessment by ultrasound. Biomed Res Int 2014: 960262, 2014.
24. Evans A, Whelehan P, Thomson K, McLean D, Brauer K, Purdie C, Baker L, Jordan L, Rauchhaus P and Thompson A: Invasive breast cancer: Relationship between shear-wave elastographic findings and histologic prognostic factors. Radiology 263: 673-677, 2012.

25. Youk JH, Gweon HM, Son EJ, Kim JA and Jeong J: Shear-wave elastography of invasive breast cancer: Correlation between quantitative mean elasticity value and immunohistochemical profile. Breast Cancer Res Treat 138: 119-126, 2013.

This work is licensed under a Creative Commons

Attribution-NonCommercial-NoDerivatives 4.0 International (CC BY-NC-ND 4.0) License. 